\title{
关于强对称和遗传对称算子 性质的几点注记
}

\author{
朱 国城 \\ (中国科学技术大学数学系,合肥)
}

\section{\$1. 引言和记号 考虑非线性发展方程}

$$
u_{t}=K(u) \text {, }
$$

这里, $u=u(x, t), K$ 是 $u$ 以及各阶微商的导数的函数. 例如当 $K(u)=6 u u_{x}+u_{x x x}$ 时, 方 程(1)就是著名的 $\mathrm{kdv}$ 方程。下画的方程

$$
\varphi_{t}=K^{\prime} \varphi
$$

称为方程 (1)的线性化方程, 这里算子 $K^{\prime}$ 定义为

$$
K^{\prime} \varphi=K^{\prime}(u)[\varphi]=\left.\frac{\partial}{\partial \varepsilon} K(u+\varepsilon \varphi)\right|_{\varepsilon=0},
$$

它是一个关于 $\varphi$ 的线性方程. $K^{\prime}$ 作为算子, 作用在 $\varphi$ 上等于 $K^{\prime}(u)[\varphi]$ ，后者称为 $K(u)$ 在 点 $\boldsymbol{u}$ 在方向 $\varphi$ 上的方向导数, 常略记为 $K^{\prime}[\varphi]$.

定义 1 一个函数 $\sigma=\sigma(u)$, 如果它是方程(2)的解, 则 $\sigma$ 称为方程 (1)的对称.

定义 2 一个算子 $\phi$ 称为方程 (1) 的强对称是指它满足

$$
\phi^{\prime}[K]-\left[K^{\prime}, \phi\right]=0,
$$

这里 $\left[K^{\prime}, \phi\right]$ 表示算子 $K^{\prime}$ 和 $\phi$ 的 Poisson 括号

$$
\left[K^{\prime}, \phi\right]=K^{\prime} \phi-\phi K^{\prime} .
$$

在文献 [1]中, 已证明强对称算子的一个很好的性质: 它把方程 (1) 的对称变为方程(1)的对 称.

定义 3 一个算子 $\phi$ 称为遗传对称 (Hereditary Symmetry)，如果对任意函数 $f$ 和 $g$, 满 足

$$
\phi^{\prime}[\phi f] g-\phi^{\prime}[\phi g] f=\phi\left\{\phi^{\prime}[f] g-\phi^{\prime}[g] f\right\},
$$

具遗传对称性质的算子有很好的性质, 反映在下述定理中 ${ }^{[1]}$.

定理 1 设 $\phi$ 是遗传对称, 又同时是方程 (1)的强对称, 则 $\phi$ 是下述方程族

$$
u_{\imath}=\phi^{m} K, m=1,2, \cdots
$$

的强对称.

\section{\$2. 强对称算子和遗传对称算子的几个性质}

现在我们来证明有关的几个引理, 最后得出一个定理.

引理 1 设 $\phi$ 是方程 (1) 的强对称, 又设 $\phi$ 可逆, 则 $\phi^{-1}$ 也是方程(1)的强对称.

本文 1985 年 6 月 18 日收到. 
证首先容易从定义出发, 验证下列式子:

$$
(\phi \psi)^{\prime}[\boldsymbol{F}]=\phi^{\prime}[\boldsymbol{F}] \psi+\phi \psi^{\prime}[\boldsymbol{F}] \text {. }
$$

特别有(取 $\psi=\phi^{-1}$ )

$$
\phi^{\prime}[\boldsymbol{F}] \phi^{-1}+\phi\left(\phi^{-1}\right)^{\prime}[\boldsymbol{F}]=0,
$$

这里的 $F$,可以是函数，也可以是算子.

现在来证明引理本身.

因为 $\phi$ 是方程 (1) 的强对称, 所以

或

$$
\begin{gathered}
\phi^{\prime}[K]-\left[K^{\prime}, \phi\right]=0, \\
\phi^{\prime}[K]-K^{\prime} \phi+\phi K^{\prime}=0 . \\
\phi^{\prime}[K] \phi^{-1}-K^{\prime}+\phi K^{\prime} \phi^{-1}=0, \\
-\phi\left(\phi^{-1}\right)^{\prime}[K]-K^{\prime}+\phi K^{\prime} \phi^{-1}=0, \\
\phi\left\{\left(\phi^{-1}\right)^{\prime}[K]+\phi^{-1} K^{\prime}-K^{\prime} \phi^{-1}\right\}=0, \\
\left(\phi^{-1}\right)^{\prime}[K]-\left[K^{\prime}, \phi^{-1}\right]=0 .
\end{gathered}
$$

右乘 $\phi^{-1}$, 得

利用(8)式, 得

或

最后得

按定义,知 $\phi^{-1}$ 是方程 (1) 的强对称.

引理 2 设 $\phi$ 是方程 (1)的强对称, 则 $\phi^{n} n=1,2, \cdots$, 也是方程 (1)的强对称.

证 只须证明如果 $\phi, \phi$ 都是方程(1)的强对称,则其乘积 $\phi \psi$ 也是方程 (1) 的强对称.

容易证明

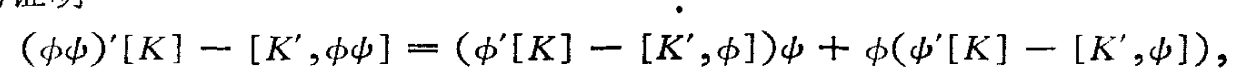

由此立得结论.

引理 3 设 $\phi$ 是遗传对称, 且 $\phi$ 可逆, 则 $\phi^{-1}$ 也是遗传对称.

证 首先, 我们需要下式, 对任意 $f, g$

$$
\phi\left(\phi^{-1}\right)^{\prime}[f] g=-\phi^{\prime}[f] \phi^{-1} g,
$$

它直接由(8)式得来.

考虑到(10)式,于是有

$$
\begin{aligned}
& \phi \cdot\left\{\left(\phi^{-1}\right)^{\prime}\left[\phi^{-1} f\right] g-\left(\phi^{-1}\right)^{\prime}\left[\phi^{-1} g\right] f\right\} \\
& =-\phi^{\prime}\left[\phi^{-1} f\right] \phi^{-1} g+\phi^{\prime}\left[\phi^{-1} g\right] \phi^{-1} f \\
& =\phi^{-1}\left\{\phi \phi^{\prime}\left[\phi^{-1} g\right] \phi^{-1} f-\phi \phi^{\prime}\left[\phi^{-1} f\right] \phi^{-1} g\right\} \\
& \stackrel{(5)}{=} \phi^{-1}\left\{\phi^{\prime}[g] \phi^{-1} f-\phi^{\prime}[f] \phi^{-1} g\right\} \\
& \stackrel{(10)}{=} \phi^{-1}\left\{-\phi\left(\phi^{-1}\right)^{\prime}[g] f+\phi\left(\phi^{-1}\right)^{\prime}[f] g\right\} \\
& =\left(\phi^{-1}\right)^{\prime}[f] g-\left(\phi^{-1}\right)^{\prime}[g] f,
\end{aligned}
$$

上式两边左乘 $\phi^{-1}$, 得到

$$
\left(\phi^{-1}\right)^{\prime}\left[\phi^{-1} f\right] g-\left(\phi^{-1}\right)^{\prime}\left[\phi^{-1} g\right] f=\phi^{-1}\left\{\left(\phi^{-1}\right)^{\prime}[f] g-\left(\phi^{-1}\right)^{\prime}[g] f\right\},
$$

按定义, 知 $\phi^{-1}$ 为遗传对称.

引理 4 设 $\phi$ 是遗传对称, 则 $\phi^{n}, n=1,2, \cdots$, 都是遗传对称.

证证明此引理,要反复运用遗传对称的性质(或定义), 即式(5).

我们要证的是下面的等式,

$$
\left(\phi^{n}\right)^{\prime}\left[\phi^{n} f\right] g-\left(\phi^{n}\right)^{\prime}\left[\phi^{n} g\right] f=\phi^{n}\left\{\left(\phi^{n}\right)^{\prime}[f] g-\left(\phi^{n}\right)^{\prime}[g] f\right\} .
$$

反复用(5)式,可得 (对任意 $k$ ) 


$$
\left(\phi^{k}\right)^{\prime}\left[\phi^{k} f\right] g=\sum_{i=0}^{k-1} \phi^{i} \phi^{\prime}\left[\phi^{k} f\right] \phi^{k-1-i} g .
$$

现在来证(11)式, 用归纳法

当 $n=1$ 时,(11)式就是(5)式,所以当 $n=1$ 时,(11)式成立.

设 $n=k-1$ 时,(11)式成立. 现考察 $n=k$ 的情形, 由(12)式可得

$$
\begin{aligned}
&\left(\phi^{k}\right)^{\prime}\left[\phi^{k} f\right] g-\left(\phi^{k}\right)^{\prime}\left[\phi^{k} g\right] f=\sum_{i=0}^{k-1} \phi^{i}\left\{\phi^{\prime}\left[\phi^{k} f\right] \phi^{k-1-i} g-\phi^{\prime}\left[\phi^{k} g\right] \phi^{k-1-i} f\right\} \\
&=\sum_{i=1}^{k-1} \phi^{i}\left\{\phi^{\prime}\left[\phi^{k} f\right] \phi^{k-1-i} g-\phi^{\prime}\left[\phi^{k} g\right] \phi^{k-1-i} f\right\} \\
&+\phi^{\prime}\left[\phi \phi^{k-1} f\right] \phi^{k-1} g-\phi^{\prime}\left[\phi \phi^{k-1} g\right] \phi^{k-1} f \\
&= \sum_{i=1}^{k-1} \phi^{i}\left\{\phi^{\prime}\left[\phi^{k} f\right] \phi^{k-1-i} g-\phi^{\prime}\left[\phi^{k} g\right] \phi^{k-1-i} f\right\} \\
&+\phi \phi^{\prime}\left[\phi^{k-1} f\right] \phi^{k-1} g-\phi \phi^{\prime}\left[\phi^{k-1} g\right] \phi^{k-1} f \\
&= \sum_{i=2}^{k-1} \phi^{i}\left\{\phi^{\prime}\left[\phi^{k} f\right] \phi^{k-1-i} g-\phi^{\prime}\left[\phi^{k} g\right] \phi^{k-1-i} f\right\} \\
&+\phi \phi^{\prime}\left[\phi^{k} f\right] \phi^{k-2} g-\phi \phi^{\prime}\left[\phi^{k} g\right] \phi^{k-2} f \\
&+\phi \phi^{\prime}\left[\phi^{k-1} f\right] \phi^{k-1} g-\phi \phi^{\prime}\left[\phi^{k-1} g\right] \phi^{k-1} f,
\end{aligned}
$$

上式最后四项的第一项与第四项, 第二项与第三项合起来使用(5)式,

$$
\begin{aligned}
\text { 原式 }= & \sum_{i=2}^{k-1} \phi^{i}\left\{\phi^{\prime}\left[\phi^{k} f\right] \phi^{k-1-i} g-\phi^{\prime}\left[\phi^{k} g\right] \phi^{k-1-i} f\right\} \\
& +\phi^{2} \phi^{\prime}\left[\phi^{k-1} f\right] \phi^{k-2} g-\phi^{2} \phi^{\prime}\left[\phi^{k-1} g\right] \phi^{k-2} f \\
& +\phi^{2} \phi^{\prime}\left[\phi^{k-2} f\right] \phi^{k-1} g-\phi^{2} \phi^{\prime}\left[\phi^{k-2} g\right] \phi^{k-1} f
\end{aligned}
$$

再取和号中 $i=2$ 的项分别与上式最后两项合起来使用(5)式, 如此继续下去, 最后可得

$$
\begin{aligned}
\text { 原式 }= & \sum_{i=2}^{k} \phi^{i}\left\{\phi^{\prime}\left[\phi^{k-1} f\right] \phi^{k-i} g-\phi^{\prime}\left[\phi^{k-1} g\right] \phi^{k-i} f\right\} \\
& +\phi^{k}\left\{\phi^{\prime}[f] \phi^{k-1} g-\phi^{\prime}[g] \phi^{k-1} f\right\} \\
= & \sum_{i=1}^{k-1} \phi^{i+1}\left\{\phi^{\prime}\left[\phi^{k-1} f\right] \phi^{k-i-1} g-\phi^{\prime}\left[\phi^{k-1} g\right] \phi^{k-i-1} f\right\} \\
& +\phi^{k}\left\{\phi^{\prime}[f] \phi^{k-1} g-\phi^{\prime}[g] \phi^{k-1} f\right\}
\end{aligned}
$$

加减相当于和号中 $i=0$ 的项,并注意到(12)式,得

$$
\begin{aligned}
{[\text { 原式= }} & \sum_{i=0}^{k-1} \phi^{i+1}\left\{\phi^{\prime}\left[\phi^{k-1} f\right] \phi^{k-i-1} g-\phi^{\prime}\left[\phi^{k-1} g\right] \phi^{k-i-1} f\right\} \\
& -\phi\left\{\phi^{\prime}\left[\phi^{k-1} f\right] \phi^{k-1} g-\phi^{\prime}\left[\phi^{k-1} g\right] \phi^{k-1} f\right\} \\
& +\phi^{k}\left\{\phi^{\prime}[f] \phi^{k-1} g-\phi^{\prime}[g] \phi^{k-1} f\right\} \\
= & \phi\left\{\left(\phi^{k}\right)^{\prime}\left[\phi^{k-1} f\right] g-\left(\phi^{k}\right)^{\prime}\left[\phi^{k-1} g\right] f\right\} \\
& -\phi\left\{\phi^{\prime}\left[\phi^{k-1} f\right] \phi^{k-1} g-\phi^{\prime}\left[\phi^{k-1} g\right] \phi^{k-1} f\right\} \\
& +\phi^{k}\left\{\phi^{\prime}[f] \phi^{k-1} g-\phi^{\prime}[g] \phi^{k-1} f\right\} \\
= & \phi\left\{\phi^{\prime}\left[\phi^{k-1} f\right] \phi^{k-1} g+\phi\left(\phi^{k-1}\right)^{\prime}\left[\phi^{k-1} f\right] g\right. \\
& \left.-\phi^{\prime}\left[\phi^{k-1} g\right] \phi^{k-1} f-\phi\left(\phi^{k-1}\right)^{\prime}\left[\phi^{k-1} g\right] f\right\}
\end{aligned}
$$




$$
\begin{aligned}
& -\phi\left\{\phi^{\prime}\left[\phi^{k-1} f\right] \phi^{k-1} g-\phi^{\prime}\left[\phi^{k-1} g\right] \phi^{k-1} f\right\} \\
& +\phi^{k}\left\{\phi^{\prime}[f] \phi^{k-1} g-\phi^{\prime}[g] \phi^{k-1} f\right\}
\end{aligned}
$$

合并, 并利用归纳假设, 可得

$$
\begin{aligned}
\text { 原式 }= & \phi^{k+1}\left\{\left(\phi^{k-1}\right)^{\prime}[f] g-\left(\phi^{k-1}\right)^{\prime}[g] f\right\} \\
& +\phi^{k}\left\{\phi^{\prime}[f] \phi^{k-1} g-\phi^{\prime}[g] \phi^{k-1} f\right\} \\
= & \phi^{k}\left\{\left(\phi^{k}\right)^{\prime}[f] g-\left(\phi^{k}\right)^{\prime}[g] f\right\},
\end{aligned}
$$

这正是要证的(11)式当 $n=k$ 时的情形,引理证毕.

由这些引理, 可证下述定理:

定理 2 设 $\phi$ 是遗传对称, 又同时是方程 (1) 的强对称, 且设 $\phi$ 可逆, 则 $\phi^{n}$ 是下述方程族

$$
u_{t}=\phi^{m} K
$$

的强对称, 且 $\phi^{n}$ 为遗传对称，这里 $m, n=0, \pm 1, \pm 2, \cdots$.

证 由所给条件和定理 1 , 知 $\phi$ 是方程族 $u_{t}=\phi^{s} K, s=0,1,2, \cdots$, 的强对称, 由引 理 $2, \phi^{l}, l=0,1,2, \cdots$ 是该方程的强对称, 再由引理 1 , 其逆 $\phi^{-l}, l=0,1,2, \cdots$, 也是该方 程的强对称. 另一方面, 由定理 2 所给条件和引理 1 知, $\phi^{-1}$ 是方程 (1) 的强对称, 又根据引 理 3 , 知 $\phi^{-1}$ 是遗传对称, 所以据定理 $1, \phi^{-1}$ 是方程族 $u_{t}=\phi^{-s} K, s=0,1,2, \cdots$ 的强对称. 由引理 $2, \phi^{-l}, l=0,1,2, \cdots$ 是方程族 $u_{t}=\phi^{-s} K, s=0.1,2, \cdots$, 的强对称. 再据引理 1 , 其逆 $\phi^{l}, l=0,1,2$ 也是此方程族的强对称. 由引理 4 知 $\phi^{n}, n=0, \pm 1, \pm 2, \cdots$ 都是遗 传对称。综上所述,即得定理 2 .

想了解更多关于强对称、遗传对称性质的读者请参阅文献 $[2,3]$.

\section{参 考 文 献}

[1] Fuchssteiner, B. and Fokas, A. S., Physica 4ID (1981), 47-66.

[2] Fuchssteiner, B., Nonlinear Anal. Theory Met!. Appl., 3(1979), 849-862.

[ 3 ] Fokas, A. S. and Fuchssteiner, B., Nonlineatr Anal. Theory Meth. Appl., 5(1981), 423-432. 